\title{
Hybrid Self-Organization Based Facility Layout Planning
}

\author{
Primož Potočnik* - Tomaž Berlec - Alojz Sluga - Edvard Govekar \\ University of Ljubljana, Faculty of Mechanical Engineering, Slovenia
}

A novel hybrid two-stage method of facility layout planning, based on self-organized clustering, is presented. In the first stage, a self-organizing map (SOM) is applied in order to organize the production process into production cells which encapsulate products with similar properties and similar machining requirements. In the second stage, the internal layout of each cell is optimized by an expert operator, taking into account various local restrictions, technological specifications, and methods of transportation. The method combines the advantages of both algorithmic and manual expert-based approaches to layout planning. The proposed method was applied in a real production company environment with promising results indicating a 42\% reduction in intensity-length efficiency measure with respect to the current layout.

Keywords: facility layout problem, self-organization, hybrid layout method, cellular manufacturing

\section{O INTRODUCTION}

Optimization of production costs in modern production can be accomplished by methods such as lean production [1] or the implementation of cellular manufacturing systems [2]. Various cell formation methods have been proposed [3] to [6], and promising results have been reported by using a self-organizing map (SOM) as a clustering tool [7] and [8]. The self-organization principle has also been proposed for facility layout planning [9] and the organization of distributed manufacturing systems [10]. Other proposed approaches for solving the cell formation and facility layout problems include partitional clustering [11], the correlation analysis approach [12], and various evolutionary, genetic and ant colony optimization algorithms [13] to [17]. An extended approach to layout planning based on the self-organization principle, which includes machines, products and the relevant attributes of products was proposed in [18].

The algorithmic approaches referenced above rely on a mathematical formulation of the facility layout problem, and provide solutions that usually do not take into account the variety of local placement restrictions with respect to size, weight, installations, technological specifications, or transportation. The algorithmic approaches thus only support human operators in creating the final layout [15]. An alternative, widely accepted approach is to apply suitable software for the manual creation and management of layout solutions [19]. Only in the case of restricted small-scale layout problems, a solution has been proposed that combines both automatic and manual modules for facility layout planning [20].

In order to combine the algorithmic formation of production cells and the manual expert-based layout planning approach, a novel hybrid two-stage facility layout planning method is proposed in this paper. The method consists of two stages: 1) the automatic selforganized formation of production cells, and 2) expert operator based fine layout planning, which finalizes the layout details. The proposed method uses the algorithmic approach in order to decompose the initial large-scale layout problem into smaller sub-problems that can be efficiently managed and solved by expert operators. The method is demonstrated on production data obtained from a manufacturing company KGL d.o.o.

The paper is organized as follows: the production data applied in this study are discussed in Section 1, whereas Section 2 presents the self-organized method for the formation of production cells, and Section 3 describes the proposed fine layout planning method. Results and discussion are presented in Section 4, and the conclusions are summarized in Section 5.

\section{PRODUCTION DATA}

The production line comprises mechanical services on $\mathrm{CNC}$ lathes and machining centres, high-pressure aluminium die casting and cast processing, the pressing of sheet metal, the fabrication of cylinders for gasoline engines, and the assembly of parts manufactured in blanks.

Operational data from the company comprise 252 products with a description of the operations required to manufacture each product, such as: band cutting, thermal cutting (laser, plasma), broaching, drilling, 3-axis CNC machining, brushing, welding, etc., and additional properties of each product as follows: materials, shape, dimensional accuracy, appearance of the product, request for examination, need for the protection of parts, weight, volume, number of assembly parts, number of operations, number of possible variants, and value. Based on the 
required operations and the additional properties, each manufactured product can be described as shown, by means of an example, in Table 1.

Table 1. An example of product description with required operations and properties

\begin{tabular}{lll}
\hline Product ID: 147035 & & \\
\hline Operations & Properties & \\
\hline 1. Band cutting & Material: & $\mathrm{Fe}$ \\
2. CNC turning & Weight: & $1.7 \mathrm{~kg}$ \\
3. Service-dip galvanizing & Volume: & $0.64 \mathrm{dm}^{3}$ \\
4. Assembly & Shape: & round \\
5. Testing & No. of parts: & 5 \\
6. Progressive compression & No. of operations: & 8 \\
7. Packaging & Dimens. accuracy: & 0.01 \\
& Appearance: & very important \\
& Examination: & functional \\
& Protection: & anticorrosion \\
& Value: & $7.81 € /$ part \\
& Quantity: & 18,000 per year \\
\hline
\end{tabular}

\subsection{Data Pre-Processing}

The product descriptions in terms of properties and required operations include various types of variables (Boolean, numerical and categorical), so the following data encoding approach was applied in order to represent the data in a numerical format suitable for automated clustering solutions:

- Boolean variables, such as the presence of a specific operation, were encoded as -1 (false) or 1 (true).

- Categorical variables (materials, form, etc.) were encoded by 1 -of-C coding that introduces additional dummy variables for each category.

- Numeric variables (weight, volume, etc.) were rescaled into a $[-1,1]$ interval.

The applied data pre-processing results in a description of each product with 58 attributes (37 operations and 21 product properties). The prepared data form a basis for self-organized cell formation and subsequent fine layout planning.

\section{SELF-ORGANIZED FORMATION OF PRODUCTION CELLS}

An important objective of the cellular organization of production is the minimization of work and material flow, and consequently the minimization of production costs. The formation of production cells can be accomplished by clustering the products according to their required operations and properties into organizational units (cells) that share similar resources. Research into clustering approaches for the organization of production cells [18] has demonstrated good properties of a SOM [21]. Compared to hierarchical clustering and k-means clustering, SOM clustering yields good clustering quality (expressed by silhouette values), and its neighbouring property guarantees the optimal arrangement of cells.

For the production data described in Section 1, hexagonal 2-dimensional SOM topology is appropriate because it corresponds to a 2-dimensional architectural layout. The decision to apply 2-dimensional topology instead of 1-diemensional one is based on the assumption that the SOM clusters should correspond to architectural arrangement of the production processes. Therefore 2-dimensional topology is better suited to floor planning. The proposed approach applies a 2-dimensional grid with $N_{c}=6$ cells (this was a recommendation from the company), arranged in 2 rows of 3 elements each, as shown in Fig. 1.

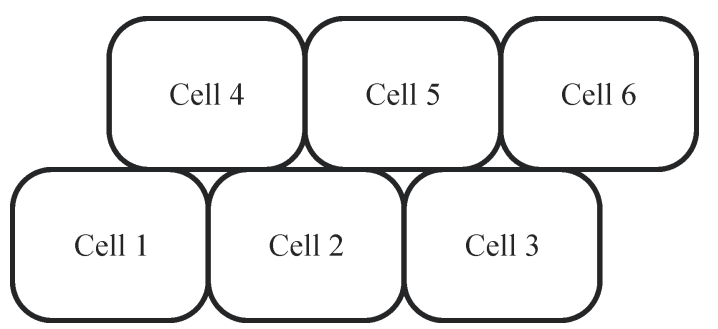

Fig. 1. Hexagonal SOM topology representing a cellular layout with 6 cells

The self-organized clustering procedure is performed by constructing the SOM clusters using both product properties and required operations. The data (properties and operations) are encoded as described in section 1.1 which results in a set of 58 attributes describing each product. In the next step, the SOM algorithm is applied to construct a mapping from this 58-dimensional space into a 2-dimensional grid representing the initial layout. The result of this stage is the initial cellular layout (Fig. 1) with products distributed in cells according to the SOM clustering algorithm.

The products in each cell share similar properties and also required operations, so the next step is to arrange the required machines into each cell. The SOM algorithm only defines which product is assigned to which cell therefore the additional interpretation of SOM results is required to define machines for each cell. The interpretation of SOM clustering results can be accomplished by examining which machines are required by products assigned to each cell $C_{i} ; i=1,2, \ldots, 6$. For each operation in a particular cell, we can provide a percentage 
of products in this cell, which should require this operation in order for this operation to be included into the production cell. Various percentile margins can be defined, such as: $p=\left\{50^{\text {th }}, 75^{\text {th }}, 100^{\text {th }}\right\}$. For each percentile margin, the particular operation should be included into the production cell if at least $(100-p) \%$ of products in a particular SOM cluster require this operation. The percentile $p$ can be considered as an open layout parameter that regulates the machine population density, i.e. the ratio of machines available in each cell and the machines that have to be borrowed in neighbouring cells.

In this study $p=75^{\text {th }}$ percentile was applied as a suitable compromise, and the result is shown in Fig. 2. For each cell $C_{i} ; i=1,2, \ldots, 6$, the assigned operations are marked in black (the columns from 1 to 37 ) and the remaining columns (38 to 58) represent the product properties. Product properties show that the products are clustered into cells also according to the similar properties, not only according to the required machine operations. Therefore the properties (columns 38 to 58) are involved in the self-organized cell formation but they do not affect the distribution of machines as described above.

The subsequent steps in arranging the SOMbased layout may include the economic optimization of each cell, and the assignment of rare operations. Economic optimization gives priority to products with high economic impact (value $\times$ quantity). Consequently, the initial placement shown in Fig. 2 can be adjusted in order to support high impact products with more required machines. The economic optimization optimizes unified percentile parameter $p$ into $p_{i} ; \mathrm{i}=1,2, \ldots, 6$, assigning different machine population densities for each cell. As first, relative economic importance of each cell is estimated by summing the economic impacts of products (value $\times$ quantity) in each cell. Then, the percentile parameter $p_{i}$ is increased in cells with high economic importance, and decreased in cells with low economic importance. The implementation of this rule for $p=75^{\text {th }}$ can be accomplished by increasing the most important cell's percentile to $p_{i}=100^{\text {th }}$, and decreasing the least important cell's percentile to $p_{i}=50^{\text {th }}$ (and in other cells linearly accordingly within this range). The result of economic optimization is shown in Fig. 3 as slightly reordered placement of machines (black operations).

Rare operations that are required to complete some products are initially not placed in the layout, so these machines are finally included in those cells with the highest requirement for such operations. The final version of the interpreted self-organized cell formation is shown in Fig. 3 with several rare operations (blue markers) included in the appropriate cells. It can be observed that several cells require the same type of machine due to frequently applied operations.

When the self-organized cell formation shown in Fig. 3 is reordered into the hexagonal floor layout shown in Fig. 1, the final version of the self-organized layout can be obtained. Fig. 4 shows such a layout with 2-dimensional cell formation, and the recommended machines in each cell. This is the result of the first stage of the proposed facility layout planning method,

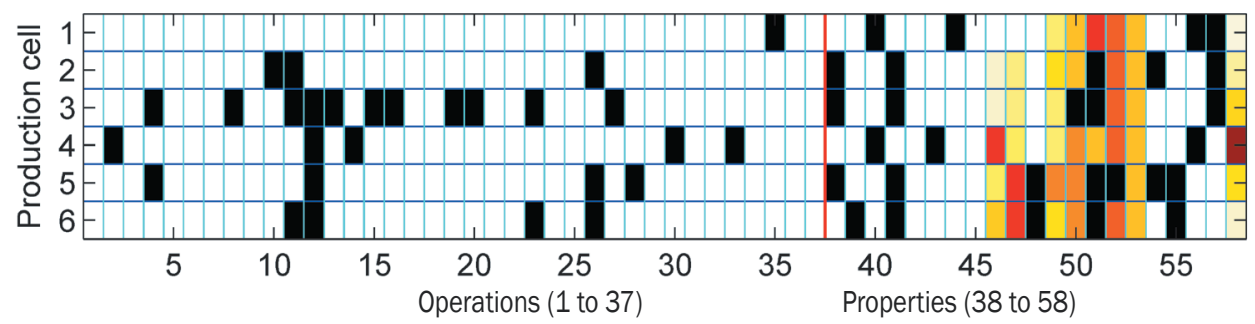

Fig. 2. Initial interpretation of SOM clusters assigning required machines to each production cell

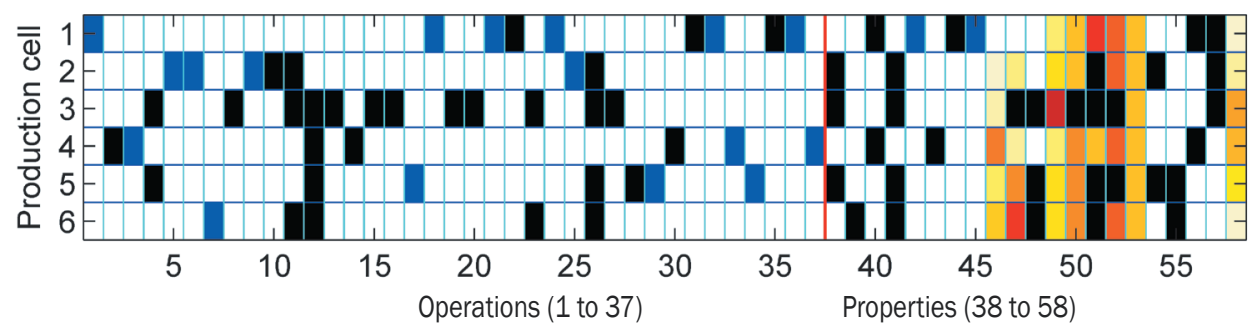

Fig. 3. Finalized interpretation of SOM clusters with an economic interpretation of the products 
i.e. automatic self-organized formation of production cells. In the next section this layout is further developed with respect to the positioning of machines within each cell.

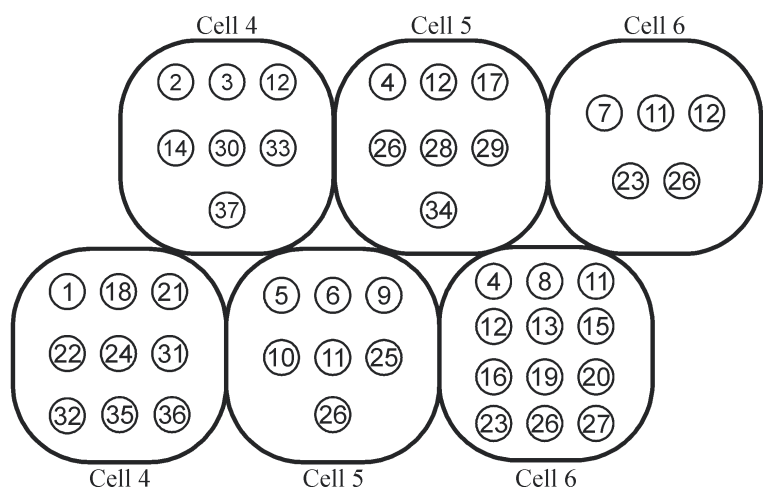

Fig. 4. Layout solution based on self-organized clustering and the economic evaluation of products

\section{FINE LAYOUT PLANNING}

Fine layout planning section describes the second stage of the proposed hybrid facility layout planning method. This stage consists of expert operator based fine layout planning that finalizes the layout details. The method proposed in this section summarizes various known approaches for layout planning but is specifically unique and tailored to complete the proposed self-organized cell formation. Because the cellular layout is already defined by self-organization, fine layout planning only considers the positioning of machines within the cells. The specificity of the proposed approach is to simultaneously optimize internal and external material flow because the selforganized cells are small but also share substantial resources with neighbouring cells in hexagonal SOM topology. An initially large-scale layout problem is decomposed into several small problems that can be efficiently solved by expert operators as described below.

\subsection{Layout Efficiency Measures}

In order to evaluate the efficiency of a layout, we propose two measures, namely total transport length $(T T L)[\mathrm{m}]$, and the product of intensity and length $(I L P)[\operatorname{part} \times \mathrm{m}]$ :

$$
\begin{gathered}
\mathrm{TTL}=\sum_{j=1}^{n} l_{j} \quad[\mathrm{~m}], \\
\mathrm{ILP}=\sum_{j=1}^{n}\left(i_{j} \cdot l_{j}\right)[\operatorname{part} \times \mathrm{m}] .
\end{gathered}
$$

The indices in Eqs. (1) and (2) denote: $n$ is the total number of paths, $l_{j}$ the transport length of the $j^{\text {th }}$ path, $i_{j}$ the intensity on the $j^{\text {th }}$ path. The total transport length can be obtained (measured) from the layout considering the sequence of operations, and then the intensity-length product can be expressed by applying the intensity values for each path. The $I L P$ measure is a weighted version of TTL because it assigns different weights to each path depending on the amount of transported items or units. When the new layout is obtained, the improvement can be measured by a relative decrease in both efficiency measures, $T T L$ and ILP.

\subsection{Material Flow Diagram and From-To Matrix}

Fine layout optimization should consider both the technology and the sequence of operations. An important tool in fine layout optimization is the material flow diagram (MFD), which can be constructed based on information about quantity, technology and the sequence of operations. The MFD shows a path of the product in a production line with the quantity transported and produced on this path. MFD is usually represented in a graphical form such as that shown in Fig. 5 for the case study presented in this paper.

Another tool in fine layout optimization is the so-called from-to matrix (FTM), which can be extracted from the MFD and presents the quantity data more clearly compared to the latter. In the FTM, all incoming pieces for each operation are collected from the MFD, which reveals how many parts pass from one operation to another.

\subsection{Graphical Layout Representation}

Layout optimization procedure usually requires a graphical representation that corresponds to the architectural layout of the production hall, and also calculates the corresponding efficiency measures for each tested layout. In our study, Vistable software was applied, although any appropriate software [19] can be used, too, for this purpose. For the case study presented in this paper, the current layout which represents the existing fine layout plan in the company is shown in Fig. 6. The efficiency of the current layout is expressed by the following values:

$$
\begin{gathered}
T T L_{\text {current }}=5933[\mathrm{~m}], \\
I L P_{\text {current }}=307 \times 10^{6}[\text { part } \times \mathrm{m}] .
\end{gathered}
$$




\subsection{Layout Creation Procedure}

Beside the described tools (layout efficiency measures, MFD, FTM, and graphical layout representation), the following additional information is required to proceed to fine layout creation:

- the current layout,

- restrictions on placement in the production hall (building restrictions, workplace restrictions, installations, technological specifications, standards and laws, etc.),

- the type of transportation,

- the standard transport routes.

The layout optimization should consider the complete workplaces and not only isolated machines, as each machine requires additional manipulating surfaces and may impose various placement restrictions with respect to size, weight, installations, technological specifications, etc. Because of the huge number of specific restrictions usually present in production environments, a mathematical model describing all the requirements and restrictions would be very difficult to construct.

Consequently, based on the tools described above, we propose an expert operator based fine layout planning approach for the finalization of the layout details. The method is applied to each cell by following the steps described below. Each step, including the optimization steps (3 and 4) can be efficiently solved by expert operator as the initial layout problem has already been decomposed into several simplified small problems.

1. Placement of cells in the production hall. The virtual space of constructed self-organized cells should be positioned within the available production space. The layout shown in Fig. 4. can be adjusted in order to fit within the production environment, and the cell orientation should

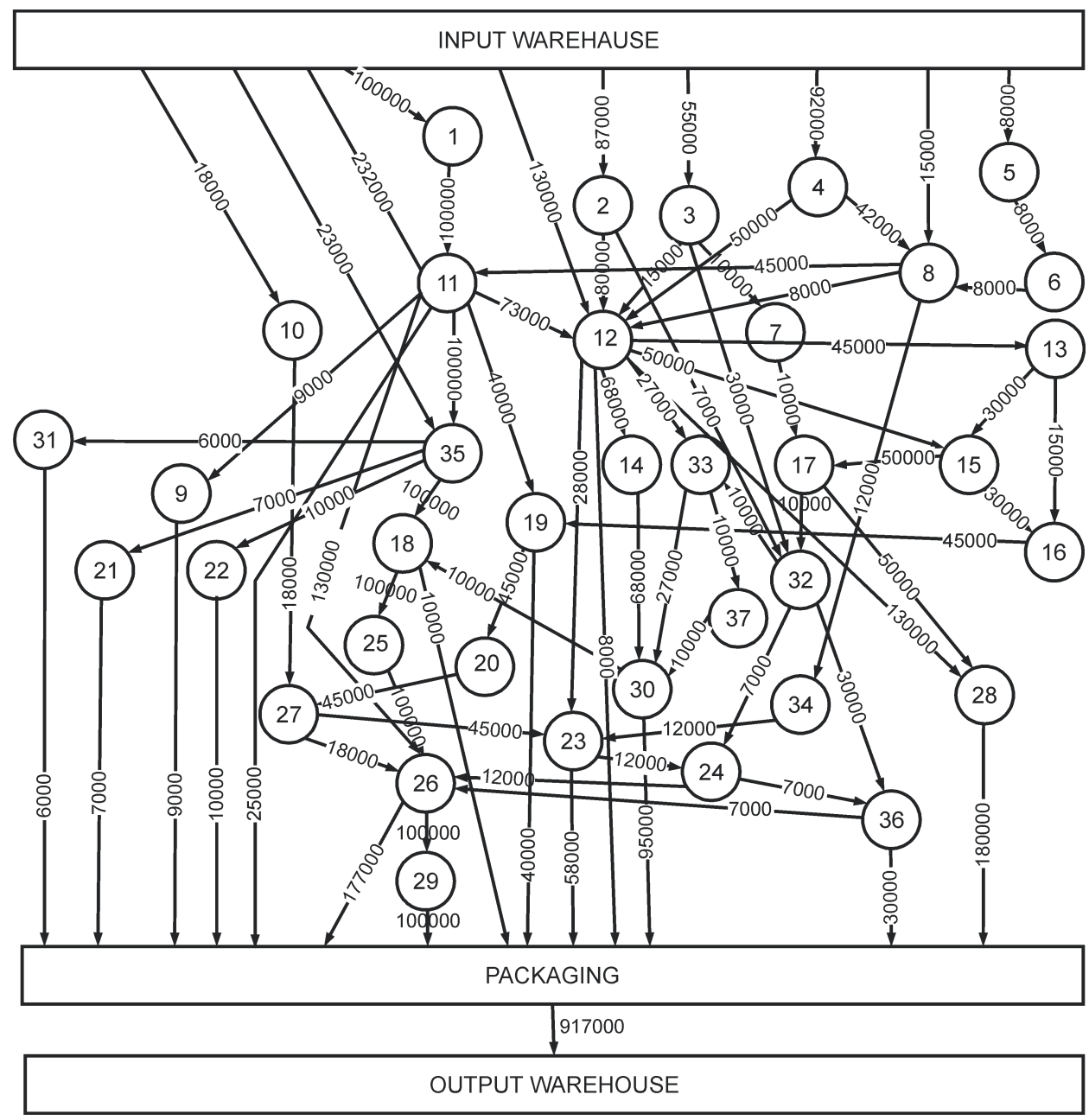

Fig. 5. Material flow diagram 


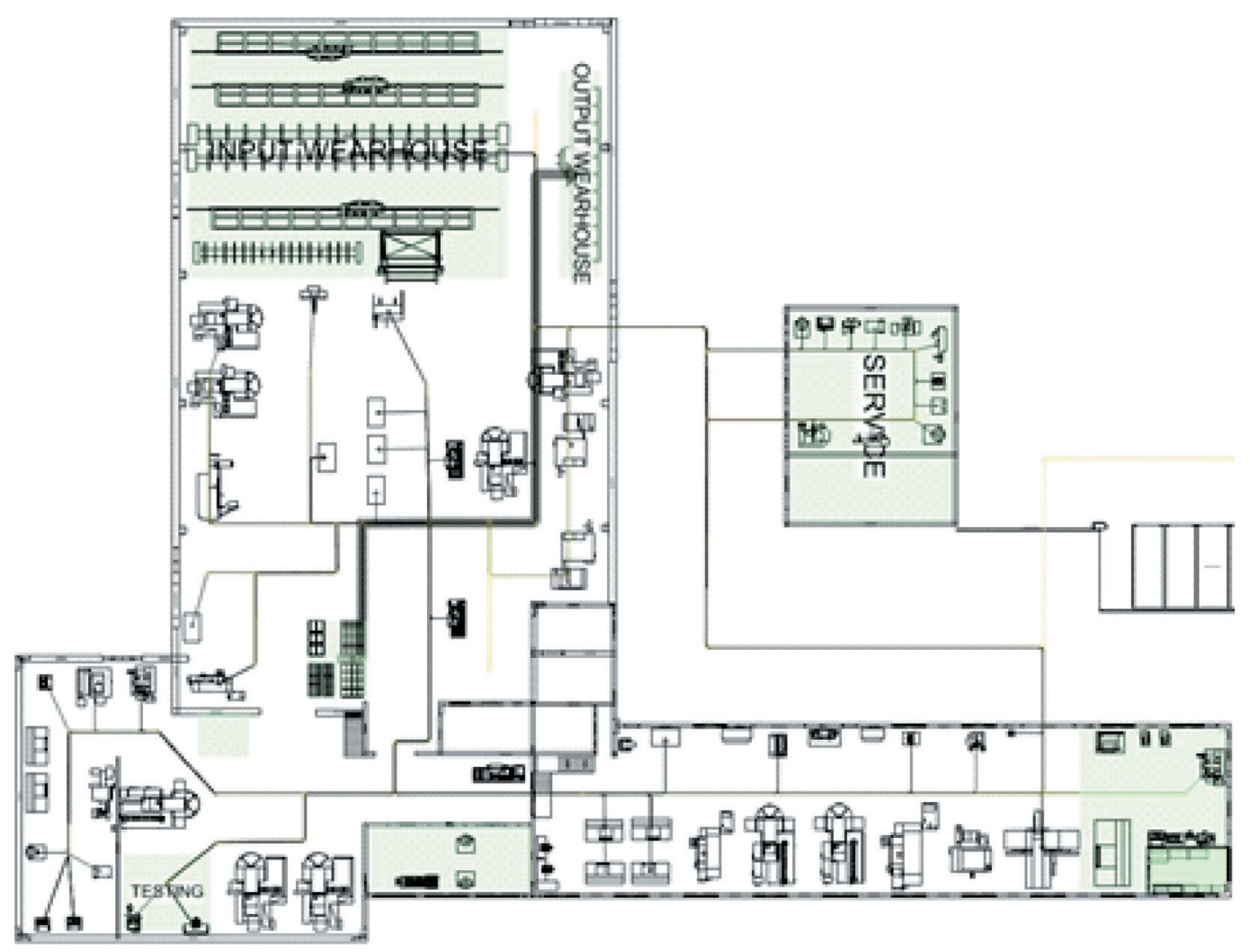

Fig. 6. Current (existing) layout

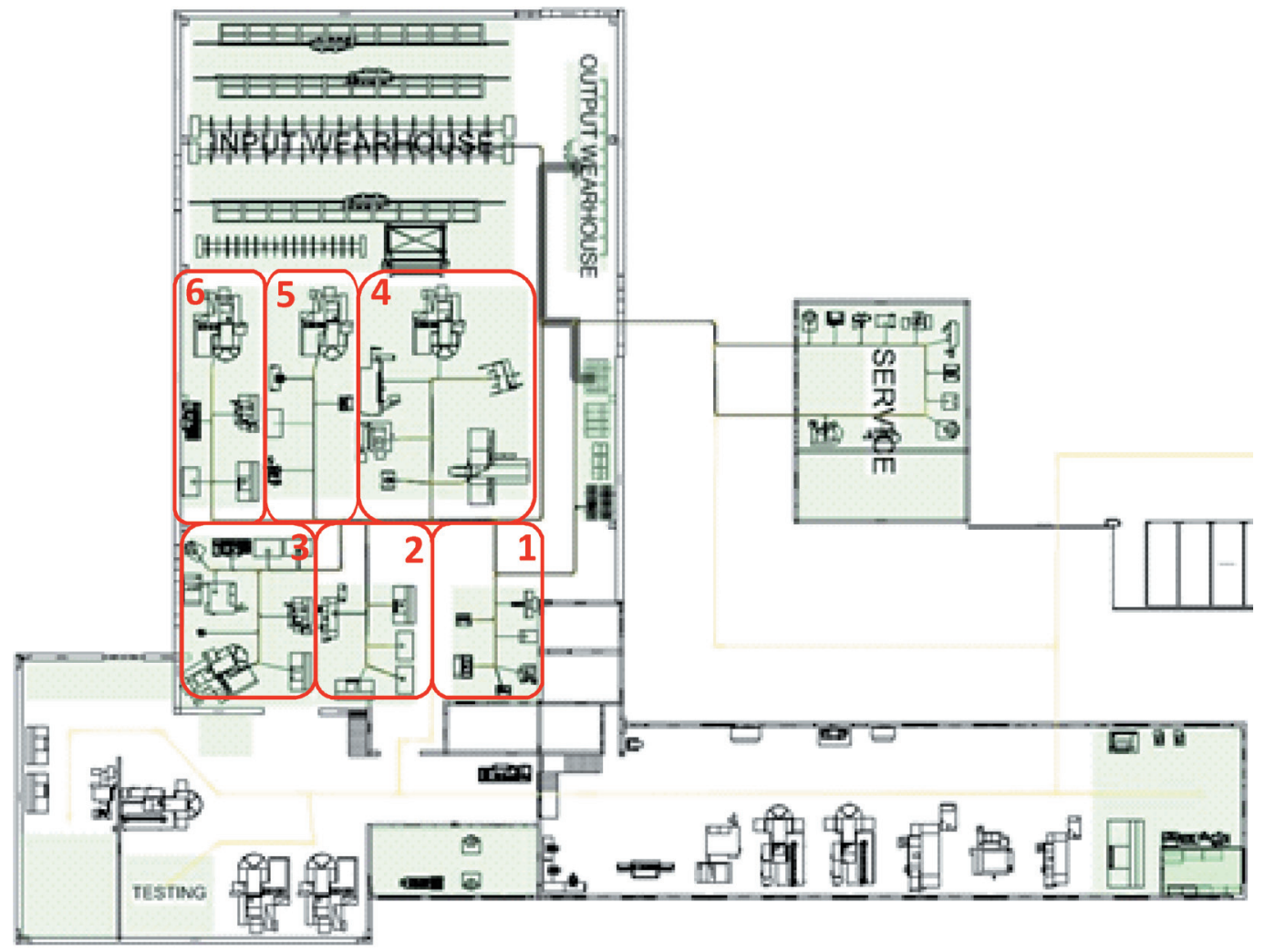

Fig. 7. The resulting layout 
minimize paths from/to the input warehouse, the packaging department, and the output warehouse.

2. Organization of workplaces within the cell. Experience showed that the optimal placement is a U-shaped cell, so an initial intra-cell layout follows a U-shaped format, with the main entrance to the cell positioned at the top of the U-shape.

3. Optimizing external material flow. From the workplaces assigned to a cell, find the two with the highest external material flow (to other cells, the warehouse, etc.). Both workplaces are positioned on opposite sides of the cell entrance, thus minimizing the external material flow to and from this cell.

4. Optimizing internal material flow. From the remaining workplaces, select the one with the highest internal material flow with respect to already positioned workplaces. Position the selected workplace according to the U-shape close to the already positioned workplaces so that the internal material flow is minimized.

5. Repeat step 4 until all the workplaces have been placed in the cell.

6. Finalize the internal layout. The expert operator should consider all the additional information (restrictions, installations, technological specifications, types of transportation, standard transport routes, etc.) and finalize the fine layout accordingly, and with respect to minimal TTL and ILP layout efficiency measures.

\section{RESULTS AND DISCUSSION}

The proposed layout creation procedure was applied to the production data considered in this study, and the resulting layout with cells marked in red is presented in Fig. 7. Evaluation of the resulting layout yielded the following efficiency:

$$
\begin{gathered}
T T L_{\text {resulting }}=3540[\mathrm{~m}], \\
I L P_{\text {resulting }}=178 \times 10^{6}[\text { part } \times \mathrm{m}] .
\end{gathered}
$$

In relative terms, the TTL and ILP measures are decreased (improved) by 40 and $42 \%$. In the presented case study, the hexagonal SOM topology with $N_{c}=6$ cells was chosen based on company demands, although $N_{c}$ can be considered as an open design parameter that may result in different layout solutions. This means that the proposed method can be adjusted to a wide range of layout planning problems with considerable flexibility. $N_{c}$ can be selected either according to the architectural conditions and/or according to the rule that the number of workplaces within each cell should be small enough to simplify the expert operator based fine layout planning. Besides the proposed hexagonal SOM topology, other topologies can be applied if they can better represent the architectural environment.

\section{CONCLUSIONS}

The paper presents a novel hybrid two-stage method for facility layout planning based on the automatic selforganized formation of production cells, and expert operator based fine layout planning. The proposed method is suitable for small and medium enterprises (SMEs) that are characterized by individual and small batch production with many different products in their production range. The method combines the advantages of both the algorithmic and the manual expert-based approaches to layout planning, and is particularly suitable for the rearrangement of existing layouts due to numerous environmental constraints. The method effectively minimizes the work and material flow, and consequently reduces production costs. The proposed approach was tested on a case study based on data provided by a real manufacturing company. The fine layout planning results show a considerable improvement in both efficiency measures, with a $40 \%$ reduction in $T T L$ and a $42 \%$ reduction in $I L P$.

\section{REFERENCES}

[1] Hines, P., Tayor, D. (2000). Going Lean. Lean Enterprise Research Centre, Cardiff Business School, Cardiff.

[2] Shishir Bhat, B.N. (2008). Cellular Manufacturing - The Heart of Lean Manufacturing. Advances in Production Engineering \& Management, vol. 3, no. 4, p. 171-180.

[3] Adenso-Díaz, B., Lozano, S., Racero J., Guerrero, F. (2001). Machine cell formation in generalized group technology. Computers \& Industrial Engineering, vol. 41, no. 2, p. 227-240, DOI:10.1016/S03608352(01)00056-0.

[4] Starbek, M., Menart, D. (2000). The optimization of material flow in production. International Journal of Machine Tools \& Manufacture, vol. 40, no. 9, p. 12991310, DOI:10.1016/S0890-6955(99)00126-1.

[5] Fung, R.Y.K., Liang, F., Jiang, Z., Wong, T.N. (2008). A multi-stage methodology for virtual cell formation oriented agile manufacturing. International Journal of Advanced Manufacturing Technologies, vol. 36, no. 7-8, p. 798-810, DOI:10.1007/s00170-006-0871-1.

[6] Fan, Z.P., Chen, Y., Ma, J., Zhu, Y. (2009). Decision support for proposal grouping: a hybrid approach using knowledge rules and genetic algorithms. Expert 
Systems with Applications, vol. 36, no. 2, p. 1004-1013, DOI:10.1016/j.eswa.2007.11.011.

[7] Kiang, M.Y., Kulkarni, U.R., Tam, K.Y. (1995). Selforganizing map network as an interactive clustering tool - An application to group technology. Decision Support Systems, vol. 15, no. 4, p. 351-374, DOI:10.1016/01679236(94)00046-1.

[8] Guerrero, F., Lozano, S., Smith, K.A., Canca, D., Kwok, T. (2002). Manufacturing cell formation using a new self-organizing neural network. Computers \& Industrial Engineering, vol. 42, no. 2-4, p. 377-382, DOI:10.1016/S0360-8352(02)00039-6.

[9] Ueda, K., Fujii, N., Hatono, I., Kobayashi, M. (2002). Facility layout planning using self-organization method. CIRP Annals - Manufacturing Technology, vol. 51, no. 1, p. 399-402, DOI:10.1016/S0007-8506(07)61546-7.

[10] Sluga, A., Butala, P., Peklenik, J. (2001). Selforganization in a distributed manufacturing system based on constraint logic programming. CIRP Annals - Manufacturing Technology, vol. 50, no. 1, p. 323-326, DOI:10.1016/S0007-8506(07)62131-3.

[11] Silva, H.B., Brito, P., da Costa, J.P. (2006). A partitional clustering algorithm validated by a clustering tendency index based on graph theory. Pattern Recognition, vol. 39, no. 5, p. 776-788, DOI:10.1016/j. patcog.2005.10.027.

[12] Hachicha, W., Masmoudi, F., Haddar, M. (2008). Formation of machine groups and part families in cellular manufacturing systems using a correlation analysis approach. The Industrial Journal of Advanced Manufacturing Technology, vol. 36, no. 11-12, p. 11571169, DOI:10.1007/s00170-007-0928-9.

[13] Chang, M., Ohkura, K., Ueda, K., Sugiyama, M. (2002). A symbiotic evolutionary algorithm for dynamic facility layout problem. Proceedings of the 2002 Congress on Evolutionary Computation, IEEE Computer Society Washington, DC, p. 1745-1750.
[14] Brintrup, A.M., Takagi, H., Ramsden, J. (2006). Evaluation of sequential, multi-objective, and parallel interactive genetic algorithms for multi-objective floor plan optimisation. Lecture Notes in Computer Science, vol. 3907, p. 586-598, DOI:10.1007/11732242_56.

[15] Ficko, M., Palčič, I. (2013). Designing a layout using the modified triangle method, and genetic algorithms. International Journal of Simulation Modelling, vol. 12, no. 4, p. 237-251, DOI:10.2507/IJSIMM12(4)3.244.

[16] Kia, R., Khaksar-Haghanib, F., Javadianc, N., Tavakkoli-Moghaddamd, R. (2014). Solving a multifloor layout design model of a dynamic cellular manufacturing system by an efficient genetic algorithm. Journal of Manufacturing Systems, vol. 33, no. 1, p. 218-232, DOI:10.1016/j.jmsy.2013.12.005.

[17] Mladineo, M., Veža, I., Čorkalo, A. (2011). Optimization of the selection of competence cells in regional production network. Tehnički vjesnik Technical Gazette, vol. 18, no. 4, p. 581-588.

[18] Potočnik, P., Berlec, T., Starbek, M., Govekar, E. (2013). Self-organizing neural network-based clustering and organization of production cells. Neural Computing and Applications, vol. 22, no. 1, p. 113-124, DOI:10.1007/s00521-012-0938-x.

[19] Shariatzadeh, N., Sivard, G., Chen, D. (2012). Software evaluation criteria for rapid factory layout planning, design and simulation, Procedia CIRP, vol. 3, p. 299304, DOI:10.1016/j.procir.2012.07.052.

[20] Jiang, S., Nee, A.Y.C. (2013). A novel facility layout planning and optimization methodology. CIRP Annals - Manufacturing Technology, vol. 62, no. 1, p. 483486, DOI:10.1016/j.cirp.2013.03.133.

[21] Kohonen, T. (1997). Self-Organizing Maps, $2^{\text {nd }}$ ed. Springer-Verlag, Berlin, DOI:10.1007/978-3-64297966-8. 